APS/123-QED

\title{
Nuclear matter incompressibility coefficient in relativistic and nonrelativistic microscopic models
}

\author{
B. K. Agrawal, S. Shlomo, and V. Kim Au \\ Cyclotron Institute, Texas A $8 M$ University, College Station, TX 77843, USA
}

\begin{abstract}
We systematically analyze the recent claim that nonrelativistic and relativistic mean field (RMF) based random phase approximation (RPA) calculations for the centroid energy $E_{0}$ of the isoscalar giant monopole resonance yield for the nuclear matter incompressibility coefficient, $K_{n m}$, values which differ by about $20 \%$. For an appropriate comparison with the RMF based RPA calculations, we obtain the parameters of the Skyrme force used in the nonrelativistic model by adopting the same procedure as employed in the determination of the NL3 parameter set of the effective Lagrangian used in the RMF model. Our investigation suggests that the discrepancy between the values of $K_{n m}$ predicted by the relativistic and nonrelativistic models is significantly less than $20 \%$.
\end{abstract}

PACS numbers: 21.65+f,24.30.Cz,21.60jz,21.10Re 


\section{INTRODUCTION}

The nuclear matter incompressibility coefficient $K_{n m}$ plays an important role in understanding a wide variety of phenomena ranging from giant resonances in finite nuclei to heavy-ion collisions and supernova explosions. The measurement of the centroid energy of the isoscalar giant monopole resonance (ISGMR) provides a very sensitive method to determine the value of $K_{n m}$. Over the last couple of decades, attempts have been made to measure very accurately the value of the ISGMR centroid energy, $E_{0}$. The recent experimental data 1] for the $E_{0}$ in heavy nuclei are accurate enough to provide unambiguous information about $K_{n m}$. However, the theoretical scenario of the $K_{n m}$ still remains unclear.

In the past, the value of $K_{n m}$ was determined using a macroscopic approach which relies on the liquid drop type of expansion for the breathing mode restoring force. It was shown that equally good fits can be obtained with values of $K_{n m}$ ranging from $100-400 \mathrm{MeV}$ 2, 3]. In other words, the liquid drop approach can not constrain the value of $K_{n m}$ better than $50 \%$. On the other hand, the microscopic determination of $K_{n m}$ using the Hartree-Fock based random phase approximation (HF-RPA) has undergone a significant improvement over time. The earlier nonrelativistic HF-RPA calculations carried out using the Skyrme interaction, which nicely reproduced the gross properties of nuclei (such as nuclear binding energy and charge radii) and the available data on isovector giant dipole resonance and isoscalar giant quadrupole resonance, yielded values of about $370 \mathrm{MeV}$ for the $K_{n m}$. With these interactions, the ISGMR in ${ }^{208} \mathrm{~Pb}$ was predicted to be located at an excitation energy of about $18 \mathrm{MeV}$. The discovery of ISGMR in ${ }^{208} \mathrm{~Pb}$ at an excitation energy of $13.7 \mathrm{MeV}$ [4] led to the modification of the Skyrme interaction. Until today, the nonrelativistic calculations with Skyrme [5, 6] and Gogny [7] interactions predict a value of $K_{n m}$ in the range of 210-220 $\mathrm{MeV}$. We remark here that the long standing problem of the conflicting results deduced for $K_{n m}$ from data on the isoscalar giant dipole resonance (ISGDR) and data on the ISGMR was explained recently by Shlomo and Sanzhur [8] as being due to a missing strength in the experimental data for the high energy region of the ISGDR.

The relativistic mean field based RPA (also referred to as RRPA) calculations, with the contribution from negative-energy sea not included, yielded for $K_{n m}$ a value in the range of $280-350 \mathrm{MeV}$ [9]. Recent RRPA calculations [10, 11], with the inclusion of negative-energy states in the response function, yield a value of $K_{n m}$ between $250-270$ 
$\mathrm{MeV}$. The discrepancy of about $20 \%$ in the value of $K_{n m}$ obtained from relativistic and nonrelativistic models is quite significant in view of the accuracy of the experimental data currently available on the ISGMR centroid energies. In recent studies [12, 13] it has been claimed that these pronounced differences are due to the model dependence of $K_{n m}$. On the other hand, it has been pointed out in Ref. [14] that the differences in the values of $K_{n m}$ obtained in the relativistic and the nonrelativistic models can be attributed, at least in part, to the differences in the density dependence of the symmetry energy in these models. However, in Ref. [14], the analysis was restricted to a single nucleus ${ }^{208} \mathrm{~Pb}$ and the interaction parameters for the several families of the effective Lagrangian considered were fitted only to the empirical values of saturation density, binding energy per nucleon in symmetric nuclear matter and the charge radius of the ${ }^{208} \mathrm{~Pb}$ nucleus. It may be pointed out here that in Ref. 15] a reasonable value of $E_{0}$ for the ${ }^{208} \mathrm{~Pb}$ nucleus was obtained using an effective force with $K_{n m}=400 \mathrm{MeV}$. But, the same effective force overestimated the value of $E_{0}$ in case of the ${ }^{90}$ Zr nucleus. Moreover, it has been suggested in Ref. [15] that a wide range of combinations of bulk, surface and asymmetry contributions to the finite nucleus compressibility can fit the energy of the ISGMR in medium to heavy nuclei. This implies that for a meaningful informations about the discrepancy between the relativistic RRPA and the nonrelativistic HF-RPA calculations, one must compare the results obtained from these models for several nuclei.

It is noteworthy that in a crude approximation, the uncertainty of about $20 \%$ in the values of $K_{n m}$ is tantamount to an uncertainty of $10 \%$ in the value of $E_{0}$. This is because, in a semi-classical approach, $E_{0} \propto \sqrt{K_{n m}}$. We have shown very recently [16] that the calculated value of $E_{0}$ can deviate by about $5 \%$ if the particle-hole space is quite limited and/or self-consistency is not properly maintained. We note that in the published literature, the calculated values of $E_{0}$ for ${ }^{208} \mathrm{~Pb}$, obtained for the same interaction, differs by up to 0.3 $\mathrm{MeV}$ [12, 17, 18]. Therefore, appropriate comparison for the values of $K_{n m}$ obtained from the different models is possible only when all the calculations are performed with the same procedure and numerical accuracy.

In this work we take a close look at the issue of the model dependence of the nuclear matter incompressibility coefficient derived from the ISGMR centroid energy. Toward this purpose, we generate different parameter sets for the Skyrme interaction and perform highly accurate calculations for the ISGMR strength function for several nuclei using the HF based 
RPA approach. For the sake of true comparison, the calculations using different parameter sets of Skyrme interaction are performed following exactly the same numerical procedure. The values of the Skyrme parameters are obtained by a least square fit to exactly the same experimental data for the nuclear binding energies, charge radii and neutron radii as adopted in Ref. 19] in determining the NL3 parameter set for an effective Lagrangian used in the RMF model. We find that the model dependence is rather weak and the differences in the values of $K_{n m}$ in the relativistic and the nonrelativistic models essentially arise from the differences in the nuclear matter properties, in particular, in the values of the symmetry energy coefficient $(J)$, associated with these models.

\section{FORMALISM}

In a self-consistent HF-RPA calculation [20], one starts by adopting a specific effective nucleon-nucleon interaction, $V_{12}$. In this work we shall use the Skyrme type interaction of the form [21],

$$
\begin{aligned}
V_{12}= & t_{0}\left(1+x_{0} P_{12}^{\sigma}\right) \delta\left(\mathbf{r}_{1}-\mathbf{r}_{2}\right)+\frac{1}{2} t_{1}\left(1+x_{1} P_{12}^{\sigma}\right) \\
& \times\left[\overleftarrow{k}_{12}^{2} \delta\left(\mathbf{r}_{1}-\mathbf{r}_{2}\right)+\delta\left(\mathbf{r}_{1}-\mathbf{r}_{2}\right) \vec{k}_{12}^{2}\right]+t_{2}\left(1+x_{2} P_{12}^{\sigma}\right) \overleftarrow{k}_{12} \delta\left(\mathbf{r}_{1}-\mathbf{r}_{2}\right) \vec{k}_{12} \\
& +\frac{1}{6} t_{3}\left(1+x_{3} P_{12}^{\sigma}\right) \rho^{\alpha}\left(\frac{\mathbf{r}_{1}+\mathbf{r}_{2}}{2}\right) \delta\left(\mathbf{r}_{1}-\mathbf{r}_{2}\right) \\
& +i W_{0} \overleftarrow{k}_{12} \delta\left(\mathbf{r}_{1}-\mathbf{r}_{2}\right)\left(\vec{\sigma}_{1}+\vec{\sigma}_{2}\right) \times \vec{k}_{12}
\end{aligned}
$$

where, $P_{12}^{\sigma}$ is the spin exchange operator, $\vec{\sigma}_{i}$ is the Pauli spin operator, $\vec{k}_{12}=-i\left(\vec{\nabla}_{1}-\vec{\nabla}_{2}\right) / 2$ and $\overleftarrow{k}_{12}=-i\left(\overleftarrow{\nabla}_{1}-\overleftarrow{\nabla}_{2}\right) / 2$. Here, right and left arrows indicate that the momentum operators act on the right and on the left, respectively. The parameters of the Skyrme force are obtained by fitting the HF results to the experimental data for the bulk properties of finite nuclei. Once the HF equations are solved using an appropriate parameter set for the Skyrme interaction, then one obtains the RPA Green's function [20]

$$
G=G_{0}\left(1+V_{p-h} G_{0}\right)^{-1},
$$

where, $V_{p-h}$ is the particle-hole $(p-h)$ interaction consistent with $V_{12}$ and $G_{0}$ is the free $p-h$ Green's function. For the single-particle operator

$$
F=\sum_{i=1}^{A} f\left(\mathbf{r}_{i}\right)
$$


the strength function is given by

$$
S(E)=\sum_{n}|\langle 0|F| n\rangle|^{2} \delta\left(E-E_{n}\right)=\frac{1}{\pi} \operatorname{Im}[\operatorname{Tr}(f G f)] .
$$

The steps involved in the relativistic mean field based RPA calculations are analogous to those described above for the nonrelativistic HF-RPA approach. However, the nucleonnucleon interaction in case of relativistic mean field models are generated through the exchange of various mesons. An effective Lagrangian which represent a system of interacting nucleons looks like [19],

$$
\begin{aligned}
\mathcal{L}= & \bar{\psi}\left(\gamma\left(i \partial-g_{\omega} \omega-g_{\rho} \vec{\rho} \vec{\tau}-e A\right)-m-g_{\sigma} \sigma\right) \psi+\frac{1}{2}(\partial \sigma)^{2} \\
& -U(\sigma)-\frac{1}{4} \Omega_{\mu \nu} \Omega^{\mu \nu}+\frac{1}{2} m_{\omega}^{2} \omega^{2}-\frac{1}{4} \vec{R}_{\mu \nu} \vec{R}^{\mu \nu} \\
& +\frac{1}{2} m_{\rho}^{2} \vec{\rho}^{2}-\frac{1}{4} F_{\mu \nu} F^{\mu \nu}
\end{aligned}
$$

which contains nucleons $\psi$ with mass $m ; \sigma, \omega, \rho$ mesons; the electromagnetic fields; and non-linear self-interactions of the $\sigma$ field,

$$
U(\sigma)=\frac{1}{2} m_{\sigma}^{2} \sigma^{2}+\frac{1}{3} g_{2} \sigma^{3}+\frac{1}{4} g_{3} \sigma^{4}
$$

The Lagrangian parameters are usually obtained, as in the case of nonrelativistic mean field calculations, by fitting procedure to some bulk properties of a set of spherical nuclei [22]. The values of various coupling constants and the meson masses appearing in Eqs. (15) and (6) for the most widely used parameter set NL3 are $m_{\sigma}=508.194 \mathrm{MeV}, m_{\omega}=782.501$ $\mathrm{MeV}, m_{\rho}=763.000 \mathrm{MeV}, g_{\sigma}=10.217, g_{\omega}=12.868, g_{\rho}=4.474, g_{2}=-10.431 \mathrm{fm}^{-1}$ and $g_{3}=-28.885$.

\section{RESULTS AND DISCUSSIONS}

\section{A. HF results}

In the present work, for an appropriate comparison, we carry out a least square fit to the same experimental data for the nuclear binding energies, charge radii and neutron radii used in Ref. 19] to obtain the NL3 parameter set. Furthermore, we deal with the centre of mass correction to the total binding energy, finite size effects of the proton and the Coulomb energy in the way similar to that employed in determining the NL3 parameter set in Ref. 
19]. It may be pointed out that pairing is not included in our HF calculations and instead of the 10 nuclei considered in Ref. 19], we consider 7 closed shell nuclei. The open shell nuclei ${ }^{58} \mathrm{Ni},{ }^{124} \mathrm{Sn}$ and ${ }^{214} \mathrm{~Pb}$ are excluded from our least square fit. We also ignore the proton and the neutron pairing gaps in case of ${ }^{90} \mathrm{Zr}$ and ${ }^{116} \mathrm{Sn}$ nuclei, respectively. However, we have verified that if we increase the error bars for the experimental data on these two nuclei in order to compensate for the pairing, we find that the values of the Skyrme interaction parameters remains practically unaltered.

Since, the main objective of this paper is to delineate the differences in the value of $K_{n m}$ predicted by the relativistic and nonrelativistic mean field based RPA calculations, we generate a Skyrme interaction having $K_{n m}$ and $J$ very close to those associated with the NL3 parameter set, i.e., 271.76 and $37.4 \mathrm{MeV}$, respectively. Also, as most of the calculation claiming the model dependence are restricted to the ISGMR centroid energy for the single heavy nucleus ${ }^{208} \mathrm{~Pb}$, we generate a parameter set by demanding a very high accuracy for the root mean square charge radius of ${ }^{208} \mathrm{~Pb}$. We denote this parameter set as SK272. In addition, we also obtain a parameter set SK255 having characteristics very much similar to the SK272 parameter set, but, $K_{n m}$ is taken to be $255 \mathrm{MeV}$.

In Table @ we give the values of the Skyrme parameters SGII together with the new parameter sets SK272 and SK255. In Table Iw we compare the nuclear matter properties for the SK272 and SK255 interactions with the corresponding ones obtained from the NL3 and SGII interactions. The quantities $\rho_{0}, m^{*} / m$ and $L$ in this table denote the saturation density, effective nucleon mass and the slope of the symmetry energy coefficient $\left(L=3 \rho_{0} d J / d \rho_{0}\right)$, respectively. In column 3 of Table III we have given the experimental data for the total binding energy, $E$, charge radii, $r_{c}$, and neutron radii, $r_{n}$, for the nuclei used in Ref. [19] for determining the NL3 parameter set and in our fit, together with the assumed error bars in percent. The values obtained from the parameter sets SK272 and SK255 are shown in columns 5 and 6, respectively. For the sake of comparison, in Table III we also give in columns 4 and 7 the results for the NL3 and SGII interactions, respectively. It is evident from this table that the quality of our fit to the experimental data is quite comparable to the results obtained with the NL3 and SGII interactions. We have also listed the values of $\Delta r=r_{n}-r_{p}$, the difference between rms radii for neutrons and protons, (not included in the fit). Experimental values for $\Delta r$ are obtained from the data for the neutron and charge radii and by using $r_{p}=\sqrt{r_{c}^{2}-0.64}$. It is interesting to note that the values of $\Delta r$ obtained 
for the SK272, SK255 and NL3 interactions are closer and are quite large compared with the corresponding results for the SGII interaction. This is consistent with the fact that the values of the slope of the symmetry energy $(L)$ associated with the SK272, SK255 and NL3 interactions are significantly larger than that associated with the SGII interaction (see Table III). Since, as is well known [23], the value of $\Delta r$ is sensitive to the density dependent form adopted for the symmetry interaction.

\section{B. RPA results}

We have demonstrated very recently [16] that the strength function for giant resonances are quite sensitive to the various numerical approximations made. By numerical approximations we essentially mean, the size of the box used for the discretization of the continuum, restriction imposed on the maximum energy for the particle-hole excitations $\left(E_{p h}^{\max }\right)$, smearing parameter $(\Gamma / 2)$ used to smear the strength function, etc. We have shown in Ref. [16] that in order to reproduce the results obtained in the continuum RPA calculation the size of the box should be consistent with the value used for the smearing width. For $\Gamma / 2=1$ $\mathrm{MeV}$, one must use a large box of size $72 \mathrm{fm}$. In this work, we have used a box of size 90 fm and $\Gamma / 2=1 \mathrm{MeV}$. From Ref. [16] we also note that in order to obtain a reliable value of the ISGMR centroid energy, $E_{0}$, accurate within $0.1 \mathrm{MeV}$, one must use $E_{p h}^{\max }>400 \mathrm{MeV}$. Here, the centroid energy is given by $E_{0}=m_{1} / m_{0}$, where $m_{0}$ and $m_{1}$ are the non-energyweighted and energy-weighted sums of $S(E)$ of Eq. (4), respectively. In the present work, the lowest value of $E_{p h}^{\max }$ we have used is higher than $500 \mathrm{MeV}$. In addition, the centroid energy depends strongly on the range adopted for the excitation energy interval of the giant resonance. Nevertheless, one often encounters in the published literature that the values of the centroid energies are given without any reference to the corresponding excitation energy range considered. For instance, in the case of the ${ }^{208} \mathrm{~Pb}$ nucleus we find for the SGII parameter set that $E_{0}=13.7,13.9,14.4 \mathrm{MeV}$ for the excitation energy ranges $0-40,0-60$ and $10-40 \mathrm{MeV}$, respectively. These differences are quite significant, since, as pointed out earlier, a variation of $5 \%$ in $E_{0}$ corresponds to a change in $K_{n m}$ by $10 \%$. In what follows, we shall concentrate mainly on the results for $E_{0}$ obtained by integrating the strength function over the energy range $0-60 \mathrm{MeV}$, since the RMF results presented in Ref. [12] for the NL3 parameter set were obtained using the same energy range and the strength function 
was smeared using $\Gamma / 2=1 \mathrm{MeV}[25]$.

In Table IV we give the results for the ISGMR centroid energy obtained using the parameter sets SK272 and SK255 and compare them with the RRPA results of Ref. [12] for the NL3 interaction. It is clear from this table that the parameter set SK272 yields values for $E_{0}$ which are higher by about $4-6 \%$ than those obtained using the NL3 parameter set in the RRPA calculations. This implies that if we reduce the compressibility by about $10 \%$, we can reproduce reasonably well the RRPA results for the NL3 interaction. For this reason we have generated another parameter set SK255 with $K_{n m}=255 \mathrm{MeV}$, keeping $J=37.4 \mathrm{MeV}$ (see Table III). In fact, we see that the differences between the values of the $E_{0}$ obtained from the parameter sets SK255 and NL3 are on the level of the uncertainty associated with the experimental data for $E_{0}$. We emphasize that though the values of $K_{n m}$ associated with the SGII and SK255 parameter sets differ by about $40 \mathrm{MeV}$, the values of $E_{0}$ for ${ }^{208} \mathrm{~Pb}$ nucleus for these interactions are close within $0.3 \mathrm{MeV}$. Thus, by requiring a fixed value of $E_{0}$ we find that an increase in $J$ by $10 \%$ leads to an increase in $K_{n m}$ by about $5 \%$. We also compute the values of $E_{0}$ over the same energy range as used in experimental determination of the centroid energy [1]. It should be noted that the experimental values of the energy range, given in the Table IV are more or less symmetric around the corresponding $E_{0}$. It can be seen from Table IV that for the parameter set SK255, we obtain a good agreement with the experimental data for $E_{0}$, calculated over the experimental excitation energy range. We remark that, in our calculations for the ${ }^{208} \mathrm{~Pb}$ nucleus with the SK272 and SK255 parameter sets, the peak energy for the isovector giant dipole resonance is 13.2 and $13.3 \mathrm{MeV}$ respectively, which is in good agreement with the experimental value of $13.3 \pm 0.1 \mathrm{MeV}$ [26].

\section{Conclusions}

In summary, we have analyzed in detail the recent claim that the nuclear matter incompressibility coefficient $K_{n m}$ extracted from the ISGMR centroid energy calculated using the relativistic and nonrelativistic based RPA models differ by about 20\%. For a meaningful comparison, we have generated parameter sets for the Skyrme interaction by a least square fitting procedure using exactly the same experimental data for the bulk properties of nuclei considered in Ref. [19] for determining the NL3 parameterization of an effective Lagrangian used in the relativistic mean field models. Further, we also demanded in our fitting proce- 
dure that the values of $K_{n m}, J$ and the charge radius of the ${ }^{208} \mathrm{~Pb}$ nucleus should be very close to the results obtained with the NL3 interaction. The parameter sets thus obtained were used to calculate the ISGMR centroid energy for several nuclei. For the parameter set SK272 $\left(K_{n m}=272 \mathrm{MeV}\right)$, the calculated values of $E_{0}$ are higher by about $5 \%$ compared to the corresponding NL3 results. This implies that the difference in the value of $K_{n m}$ obtained in the relativistic and the nonrelativistic microscopic models could be at most $10 \%$. In view of this, we generate another parameter set having $K_{n m}=255 \mathrm{MeV}$. As expected, the parameter set associated with $K_{n m}=255 \mathrm{MeV}$, yields for the ISGMR centroid energies values which are quite close to the NL3 results. Moreover, for the SK255 parameter set, we find a good agreement with experimental data for $E_{0}$ for all the nuclei considered, provided, the corresponding excitation energy ranges used in determining $E_{0}$ are the same as those used in obtaining the experimental data. We have thus shown that the difference in the values of $K_{n m}$ obtained in the relativistic and nonrelativistic models is mainly due to the differences in the values of the symmetry energy coefficient $(J)$ and its slope $(L)$ associated with these models.

[1] D. H. Youngblood, H. L. Clark and Y. W. Lui, Phys. Rev. Lett. 82, 691 (1999).

[2] J. M. Pearson, Phys. Lett. B271, 12 (1991).

[3] S. Shlomo and D. H. Youngblood, Phys. Rev. C 47, 529, (1993).

[4] N. Marty, et al, Nucl. Phys. A230, 93 (1975).

[5] G. Colo, P. F. Bortignon, N. Van Giai, A. Bracco and R. A. Broglia, Phys. Lett. B276, 279 (1992).

[6] I. Hamamoto, H. Sagawa and H. Z. Zhang, Phys. Rev. C 56, 3121 (1997).

[7] J. P. Blaizot, J. F. Berger, J. Decharge and N. Girod, Nucl. Phys. A591, 435 (1995).

[8] S. Shlomo and A. I. Sanzhur, Phys. Rev. C 65, 044310 (2002); S. Shlomo, Pramana-J. 57, $557(2001)$.

[9] Zhongyu Ma, Nguyen Van Giai and Hiroshi Toki, Phys. Rev. C 55, 2385 (1997).

[10] Zhong-yu Ma, Nguyen Van Giai, A. Wandelt, D. Vretenar, Nucl. Phys. A686, 173 (2001).

[11] D. Vretenar, T. Niksic and P. Ring, Phys. Rev. C, in press.

[12] Nguyen Van Giai, P. F. Bortignon, G. Colo, Zhongyu Ma and M. Quaglia, Nucl. Phys. A687, 
44c (2001).

[13] T. Niksic, D. Vretenar and P. Ring, Phys. Rev. C 66, 064302 (2002).

[14] J. Piekarewicz, Phys. Rev. C 66, 034305 (2002).

[15] F. E. Serr, Proc. Nuclear Physics Workshop, Trieste (Italy), October 1981, eds. C. H. Dasso, R. A. Broglia and A. Winthelr (North-Holland, Amsterdam, 1982).

[16] B. K. Agrawal, S. Shlomo and A. I. Sanzhur, Phys. Rev. C 67, 034114 (2003).

[17] Gianluca Colo, Nguyen Van Giai Pier F. Bortignon and Maria R. Quaglia, RIKEN Review No. 23 (July 1999).

[18] Gianluca Colo, Nguyen Van Giai Pier F. Bortignon and Maria R. Quaglia, Physics of Atomic Nuclei Vol. 64, No. 6, 1044 (2001).

[19] G. A. Lalazissis, J. Konig and P. Ring, Phys. Rev. C 55, 540 (1997).

[20] G. F. Bertsch and S. F. Tsai, Phys. Rep. 18, 125 (1975).

[21] D. Vautherin and D. M. Brink, Phys. Rev. C 5, 626, (1972).

[22] P. -G. Reinhard, Rep. Prog. Phys. 52, 439 (1989).

[23] E. Friedman and S. Shlomo, Z. Physik A283, 67 (1977).

[24] Nguyen Van Giai and H. Sagawa, Phys. Lett. B106, 379 (1981).

[25] Zhong-yu Ma, private communication.

[26] J. Ritman et al., Phys. Rev. Lett. 70, 533 (1993). 
TABLE I: Skyrme parameters for different interactions used in the present calculations. Value of the parameters for the SGII interaction are taken from Ref. [24].

\begin{tabular}{|cccc|}
\hline Parameter & SK272 & SK255 & SGII \\
\hline$t_{0}\left(\mathrm{MeV} \cdot \mathrm{fm}^{3}\right)$ & -1496.84 & -1689.35 & -2645 \\
$t_{1}\left(\mathrm{MeV} \cdot \mathrm{fm}^{5}\right)$ & 397.66 & 389.30 & 340 \\
$t_{2}\left(\mathrm{MeV} \cdot \mathrm{fm}^{5}\right)$ & -112.82 & -126.07 & -41.9 \\
$t_{3}\left(\mathrm{MeV} \cdot \mathrm{fm}^{3(1+\alpha)}\right)$ & 10191.64 & 10989.60 & 15595 \\
$x_{0}$ & 0.0008 & -0.1461 & 0.09 \\
$x_{1}$ & 0.0102 & 0.1160 & -0.0588 \\
$x_{2}$ & 0.0020 & 0.0012 & 1.425 \\
$x_{3}$ & -0.5519 & -0.7449 & 0.06044 \\
$a l p h a$ & 0.4492 & 0.3563 & $1 / 6$ \\
$W_{0}\left(\mathrm{MeV} \cdot \mathrm{fm}^{5}\right)$ & 106.58 & 95.39 & 105 \\
\hline
\end{tabular}

TABLE II: Nuclear matter properties calculated from RMF theory with the NL3 parameter set and the nonrelativistic HF calculations with different Skyrme parameter sets. The "experimental data" are the ones used in Ref. [19] in the least square fit together with the bulk properties for finite nuclei in obtaining the NL3 parameter set. The values in parenthesis represent the error bars (in percent) used in the fit.

\begin{tabular}{|cccccc|}
\hline & Exp. & NL3 & SK272 & SK255 & SGII \\
\hline$E / A(\mathrm{MeV})$ & $-16.0(5)$ & -16.299 & -16.280 & -16.334 & -15.67 \\
$K_{n m}(\mathrm{MeV})$ & $250.0(10)$ & 271.76 & 271.55 & 254.96 & 214.57 \\
$\rho_{0}\left(\mathrm{fm}^{-3}\right)$ & $0.153(10)$ & 0.148 & 0.155 & 0.157 & 0.159 \\
$m^{*} / m$ & & 0.60 & 0.77 & 0.80 & 0.79 \\
$J(\mathrm{MeV})$ & $33.0(10)$ & 37.4 & 37.4 & 37.4 & 26.8 \\
$L(\mathrm{MeV})$ & & 118.5 & 91.7 & 95.0 & 37.6 \\
\hline
\end{tabular}


TABLE III: Experimental data for the total binding energy $(E)$ in $\mathrm{MeV}$, charge $\left(r_{c}\right)$ and neutron $\left(r_{n}\right)$ radii in fm for the nuclei used in the least square fitting procedure. The parameter sets thus obtained are named as SK272 and SK255. For comparison we also give the results obtained from the NL3 and SGII interactions. The values in parenthesis represent the error bars (in percent) used in the fit. The quantity $\Delta r=r_{n}-r_{p}$ (not included in the fit) is the difference between the radii for the neutrons and protons.

\begin{tabular}{|c|c|c|c|c|c|c|}
\hline Nucleus & coperty & Exp. & NL3 & SK272 & SK255 & SGII \\
\hline \multirow[t]{3}{*}{${ }^{16} \mathrm{O}$} & $E$ & $-127.62(0.1)$ & -128.83 & -127.76 & -128.05 & -131.93 \\
\hline & $r_{c}$ & $2.730(0.2)$ & 2.730 & 2.800 & 2.813 & 2.793 \\
\hline & $r_{n}$ & & 2.580 & 2.662 & 2.674 & 2.650 \\
\hline \multirow[t]{4}{*}{${ }^{40} \mathrm{Ca}$} & $E$ & $-342.06(0.1)$ & -342.02 & -341.35 & -342.50 & -342.42 \\
\hline & $r_{c}$ & $3.450(0.2)$ & 3.469 & 3.496 & 3.504 & 3.490 \\
\hline & $r_{n}$ & $3.370(2.0)$ & 3.328 & 3.363 & 3.369 & 3.348 \\
\hline & $\Delta r$ & 0.014 & -0.047 & -0.041 & -0.043 & -0.049 \\
\hline \multirow[t]{4}{*}{${ }^{48} \mathrm{Ca}$} & $E$ & $-416.00(0.1)$ & -415.15 & -414.17 & -413.89 & -418.22 \\
\hline & $r_{c}$ & $3.451(0.2)$ & 3.470 & 3.524 & 3.531 & 3.526 \\
\hline & $r_{n}$ & $3.625(2.0)$ & 3.603 & 3.635 & 3.649 & 3.582 \\
\hline & $\Delta r$ & 0.268 & 0.227 & 0.203 & 0.210 & 0.147 \\
\hline \multirow[t]{4}{*}{${ }^{90} \mathrm{Zr}$} & $E$ & $-783.90(0.1)$ & -782.63 & -782.73 & -783.28 & -775.49 \\
\hline & $r_{c}$ & $4.258(0.2)$ & 4.287 & 4.282 & 4.286 & 4.286 \\
\hline & $r_{n}$ & $4.289(2.0)$ & 4.306 & 4.310 & 4.317 & 4.266 \\
\hline & $\Delta r$ & 0.107 & 0.094 & 0.103 & 0.106 & 0.056 \\
\hline \multirow[t]{4}{*}{${ }^{116} \mathrm{Sn}$} & $E$ & $-988.69(0.1)$ & -987.67 & -982.37 & -984.48 & -971.66 \\
\hline & $r_{c}$ & $4.627(0.2)$ & 4.611 & 4.617 & 4.619 & 4.630 \\
\hline & $r_{n}$ & $4.692(2.0)$ & 4.735 & 4.696 & 4.701 & 4.639 \\
\hline & $\Delta r$ & 0.135 & 0.194 & 0.149 & 0.152 & 0.079 \\
\hline \multirow[t]{3}{*}{${ }^{132} \mathrm{Sn}$} & $E$ & $-1102.90(0.1)$ & -1105.44 & -1097.36 & 1100.04 & -1105.17 \\
\hline & $r_{c}$ & & 4.709 & 4.725 & 4.726 & 4.735 \\
\hline & $r_{n}$ & & 4.985 & 4.964 & 4.975 & 4.867 \\
\hline \multirow[t]{4}{*}{${ }^{208} \mathrm{~Pb}$} & $E$ & $-1636.47(0.1)$ & -1639.54 & -1631.78 & -1637.48 & -1622.21 \\
\hline & $r_{c}$ & $5.503(0.2)$ & 5.520 & 5.503 & 5.503 & 5.519 \\
\hline & $r_{n}$ & $5.593(2.0)$ & 125.741 & 5.687 & 5.694 & 5.597 \\
\hline & $\Delta r$ & 0.148 & 0.279 & 0.243 & 0.250 & 0.136 \\
\hline
\end{tabular}


TABLE IV: ISGMR centroid energy $E_{0}=m_{1} / m_{0}$ (in MeV) obtained by integrating over the energy range $\omega_{1}-\omega_{2} \mathrm{MeV}$ with the strength function smeared by using $\Gamma / 2=1 \mathrm{MeV}$. The experimental data is taken from Ref. 1].

\begin{tabular}{|c|c|c|c|c|c|c|}
\hline \multicolumn{2}{|c|}{ Nucleus $\omega_{1}-\omega_{2}$} & \multirow[t]{2}{*}{ Exp. } & \multicolumn{2}{|c|}{ NL3 SK272 } & SK 255 & SGII \\
\hline \multirow[t]{2}{*}{${ }^{90} \mathrm{Zr}$} & $0-60$ & & 18.7 & 20.0 & 18.9 & 18.3 \\
\hline & $10-26$ & $7.89 \pm 0.20$ & & 19.3 & 18.4 & 17.9 \\
\hline \multirow[t]{2}{*}{${ }^{116} \mathrm{Sn}$} & $0-60$ & & 17.1 & 18.0 & 17.5 & 16.6 \\
\hline & $10-23$ & $6.07 \pm 0.12$ & & 17.4 & 16.9 & 16.3 \\
\hline \multirow[t]{2}{*}{${ }^{144} \mathrm{Sm}$} & $0-60$ & & 16.1 & 17.1 & 16.4 & 15.6 \\
\hline & $10-22$ & $5.39 \pm 0.28$ & & 16.5 & 15.9 & 15.2 \\
\hline \multirow[t]{2}{*}{${ }^{208} \mathrm{~Pb}$} & $0-60$ & & 14.2 & 14.7 & 14.2 & 13.9 \\
\hline & $8-21$ & $4.17 \pm 0.28$ & & 14.2 & 13.8 & 13.6 \\
\hline
\end{tabular}

